\title{
Risk factors for delirium after coronary artery bypass grafting in elderly patients
}

\author{
Jian $\mathrm{Li}^{1}$, Dongmei Meng ${ }^{1}$, Chao Chang ${ }^{1}$, Bo Fu ${ }^{2}$, Chang Xie ${ }^{1}$, Zhenhua Wu ${ }^{1}$, Lianqun Wang ${ }^{2}$ \\ ${ }^{1}$ Intensive Care Unit, Tianjin Chest Hospital, Tianjin, China; ${ }^{2}$ Department of Cardiovascular Surgery, Tianjin Chest Hospital, Tianjin, China \\ Contributions: (I) Conception and design: J Li, Z Wu; (II) Administrative support: L Wang; (III) Provision of study materials or patients: J Li, D \\ Meng; (IV) Collection and assembly of data: B Fu, C Xie; (V) Data analysis and interpretation: C Chang; (VI) Manuscript writing: All authors; (VII) \\ Final approval of manuscript: All authors. \\ Correspondence to: Professor Zhenhua Wu, MD. Intensive Care Unit, Tianjin Chest Hospital, No. 261, South Taierzhuang Road, Tianjin 300051, \\ China. Email: wzh8306@aliyun.com; Professor Lianqun Wang, MD. Department of Cardiovascular Surgery, Tianjin Chest Hospital, No. 261, South \\ Taierzhuang Road, Tianjin 300051, China. Email: lianqun1964@hotmail.com.
}

Background: Postoperative delirium (POD) is a common complication of major surgery and is associated with fortified morbidity, mortality, and long-term cognitive dysfunction. This study sought to evaluate the incidence and risk factors of delirium in elderly (aged $\geq 65$ years) patients who underwent coronary artery bypass grafting (CABG).

Methods: We performed a retrospective cohort analysis. The clinical data of 1,426 elderly patients who underwent CABG at our hospital from October 2018 to October 2020 were collected and analyzed. Delirium was defined as any positive Confusion Assessment Method for Intensive Care Unit examination following surgery during the intensive care unit (ICU) stay. Risk factors for POD were authenticated via univariate and multivariate logistic regression analyses. The intraoperative and postoperative factors were evaluated using a propensity score-matched regression analysis based on preoperative factors.

Results: A total of $39.3 \%$ (560 of 1,426) of elderly patients who underwent CABG were diagnosed with delirium. Based on the multivariate analysis, age [odds ratio (OR) 1.013], long-term alcohol consumption (OR 2.026), diabetes (OR 1.51), stroke (OR 1.41), and extracardiac arteriopathy (OR 1.61) were found to be independent predictors of post-cardiac surgery delirium. Conversely, high-density lipoprotein cholesterol levels (HDL-C) $\geq 1.0 \mathrm{mmol} / \mathrm{L}$ (OR 0.71) was found to be a protective factor. Among the intraoperative and postoperative factors evaluated, only a prolonged ICU stay ( $\geq 48 \mathrm{~h}$; OR 1.62) was identified as a risk factor for developing delirium in the propensity-score matched analysis, after adjusting for potential confounding variables and selection bias. In contrast to the earlier analysis, the multivariate logistic regression further revealed that peri-procedural myocardial infarction and postoperative arterial oxygen partial pressures were not significantly associated with POD.

Conclusions: The incidence of POD in elderly patients undergoing CABG was high. Factors such as older age, long-term alcohol consumption, diabetes, stroke, and extracardiac arteriopathy were independent risk factors for POD in these patients. In relation to the intraoperative and postoperative factors, after adjusting for preoperative confounding factors, only a prolonged ICU stay was identified as a risk factor for POD. Additionally, high levels of HDL-C may be beneficial in reducing the incidence of delirium.

Keywords: Elderly; delirium; risk factors; coronary artery bypass grafting (CABG)

Submitted Sep 09, 2021. Accepted for publication Nov 03, 2021.

doi: $10.21037 / \mathrm{atm}-21-5160$

View this article at: https://dx.doi.org/10.21037/atm-21-5160 


\section{Introduction}

Postoperative delirium (POD), an acute and fluctuating disorder of attention and cognition (1), is a common complication after anesthesia and surgery. POD can occur in patients of any age, and its incidence is significantly influenced by patient-related risk factors and type of surgery. It is generally believed that the risk of elderly patients is higher, because the preoperative complications, organ dysfunction, malnutrition and other risk factors are accumulated and superimposed with age (2). It is reported that the incidence of POD is the highest in elderly patients undergoing cardiac surgery and non-cardiac major surgery (3).

Coronary artery bypass grafting $(\mathrm{CABG})$ is an important technique for coronary atherosclerotic heart disease and is one of the most commonly performed cardiac surgeries $(4,5)$. CABG effectively improves coronary blood supply and cardiac function, and has a low mortality rate (6). In different studies, reports on the incidence of delirium after CABG have varied; however, all the results suggest that the development of delirium has severe consequences for patients, such as a longer hospital stay, a higher risk of mortality, and long-term decreases in overall function, cognitive function, and quality of life (7-10). Knowledge of the incidence of delirium and identification of protective and risk factors will inform resource allocation and direct future studies.

At present, the pathogenesis of delirium is unclear, genetic research of delirium was limited and indicating conflicting results (11-13). Researchers tried to find simple and meaningful factors to timely diagnose as well as correctly predict POD. More importantly, it was expected that the incidence of POD could be reduced by changing some variable risk factors. A prospective cohort study (14) about 249 Chinese patients who underwent CABG showed that the incidence of POD was $30.52 \%$, and the factors independently associated with delirium were preoperative atrial fibrillation, elevated European system for cardiac operative risk evaluation and cognitive impairment, longer surgery duration, postoperative poor quality of sleep, and electrolyte disturbance. Lechowicz et al. suggested that an index composed of age, preoperative level of glycated hemoglobin (HbA1c), and preoperative platelet-to-white blood cell ratio (PWR), could be clinically useful for prediction of POD after CABG (8).

Numerous studies (15-17) have shown that age is one of the main risk factors for delirium after cardiac surgery. Despite the enhanced interest in delirium after CABG, very few studies have specifically focused on older patients, and the number of patients included in previous studies has been relatively small. Thus, this study sought to examine the morbidity of delirium in elderly patients undergoing CABG, and more specifically, to confirm and verify intraoperative, preoperative, and postoperative risk factors of intensive care unit (ICU) delirium after CABG. We present the following article in accordance with the STROBE reporting checklist (available at https://dx.doi.org/10.21037/atm-21-5160).

\section{Methods}

\section{Patients}

We performed a retrospective cohort analysis of data from 1,426 elderly (aged $\geq 65$ years) patients of both sexes who underwent isolated CABG at our hospital from October 2018 to October 2020. In accordance with the definition of the World Health Organization, most developed countries in the world define elderly as being aged $\geq 65$ years. The patients in this study included 1,023 men $(71.72 \%)$, aged 65-92 years (mean: 71.28 4.768 years).

Patients aged $\geq 65$ years who underwent isolated CABG, whether under cardiopulmonary bypass or off-pump, were included in this study. Patients were excluded from this study if they met any of the following exclusion criteria were: (I) had a preoperative cognitive disorder (cognitive impairment, delirium, or dementia); (II) had primary mental maladjustments; (III) had a language or hearing impairment, coma, or end-stage disease; (IV) could not complete the POD assessment; (V) died within the first $24 \mathrm{~h}$ after the operation or intraoperatively; and/or (VI) had incomplete or inaccurate clinical data. The research was authorized by the Ethics Committee of Tianjin Chest Hospital (No. 2021LW-012). All the procedures performed in this study involving human participants were conducted in accordance with the Declaration of Helsinki (as revised in 2013). The requirement of personal consent for this retrospective analysis was waived.

\section{Operative procedure and data collection}

Potential risk factors were selected based on our database and the existing literature. Demographic data, preoperative data, comorbidities, the process of operation, and postoperative outcomes were extracted from patients' medical records. The methods for anesthesia, preoperative evaluation and medication, and intraoperative procedures 
were all performed in accordance with well-ascertained local guidelines. Laboratory data outcomes were taken from standard preoperative and postoperative blood sampling. Surgeries included all off-pump and on-pump CABG performed under general anesthesia.

\section{Assessment of delirium}

Patients' mental status data were obtained from their intensive care records. POD, which excluded agitation during the recovery period, was assessed once per 12-h shift using the Confusion Assessment Method for Intensive Care Unit (CAM-ICU) examination during the ICU stay. The nurses at our institution have received extensive training on how to complete accurate CAM-ICU examinations at the bedside.

\section{Statistical analysis}

The delineated statistics were used to describe the research population. Commonly distributed continuous variables are presented as the mean and standard deviation, and were evaluated using the independent samples $t$-test. Skewed continuous variables are presented as the median and interquartile ranges, and were evaluated using the MannWhitney U-test. Categorical variables are presented as frequencies with percentages and were evaluated using the chi-square test or Fisher's exact test for small sample sizes. Univariate and multivariate logistic regression analyses of intraoperative, preoperative, and postoperative factors related to POD were conducted, and odds ratios (ORs) with $95 \%$ confidence intervals were calculated.

To balance the differences in preoperative baseline variables between the two groups with and without delirium, propensity-score matching (PSM) was performed based on a logistic regression model containing the following covariates: age, sex, body mass index, longterm drinking ( $>10$ years), smoking, diabetes mellitus, arterial hypertension, chronic renal disease, stroke, chronic obstructive pulmonary disease, internal carotid artery (ICA) stenosis, old myocardial infarction, congestive heart failure, atrial fibrillation, pulmonary hypertension, laboratory data, and ejection fraction (EF) before surgery. Cases were matched in a 1:1 fashion using the nearest-neighbor principle with a caliper of 0.2 . The quality of matching was assessed visually using a density plot of the propensity score. Statistical significance $(\mathrm{P})$ was set at $<0.05$. The software program IBM SPSS statistics version 23.0 (IBM SPSS
Statistics, RRID:SCR_019096) and the free R4.0.5 software package were used for the statistical analysis and habit-score matching.

\section{Results}

Determined by the number of cases in the center during the study period, we analyzed a cohort of 3,992 patients and included 1,426 (35.72\%) elderly patients who underwent CABG in the final analysis. Of the patients, 560 of 1,426 (39.27\%) were diagnosed with delirium.

\section{Baseline patient characteristics}

We collected relevant data of each patient during hospitalization, the average length was $16.18 \pm 5.79$ days. The preoperative characteristics of the elderly patients who underwent CABG are shown in Table 1. Patients with delirium were older, more often diabetic, had a history of long-term drinking, stroke, ICA stenosis, and had a higher serum total cholesterol, low-density lipoprotein cholesterol (LDL-C), and lower high-density lipoprotein cholesterol (HDL-C), and EF than their counterparts without delirium $(\mathrm{P}<0.05)$.

The postoperative features of patients are presented in Table 2. Cardiopulmonary bypasses and intra-aortic balloon pumps were more frequently used in patients with POD than in patients without POD. In relation to the postoperative complications after CABG, low cardiac output syndrome, perioperative myocardial infarction (PMI), cardiac arrest or ventricular fibrillation, pneumonia, and multiple organ dysfunction syndrome occurred more often in elderly patients with delirium than in elderly patients without delirium. Postoperative serum creatinine was higher and arterial oxygen partial pressure $\left(\mathrm{PO}_{2}\right)$ was lower in elderly patients with delirium than in those without delirium. The length of stay (LOS) in the ICU and the duration of mechanical ventilation were longer for patients with delirium than for those without delirium $(\mathrm{P}<0.05)$.

\section{Univariate and multivariate analyses of POD in elderly CABG patients}

The risk factors for POD in the entire cohort were analyzed. Some consecutive variables were changed into classified variables and were included in the regression model. The grouping methods and bases are set out in Table 3 . The outcomes of the univariate analysis and multivariable 
Table 1 Preoperative characteristics of elderly patients with and without delirium after CABG

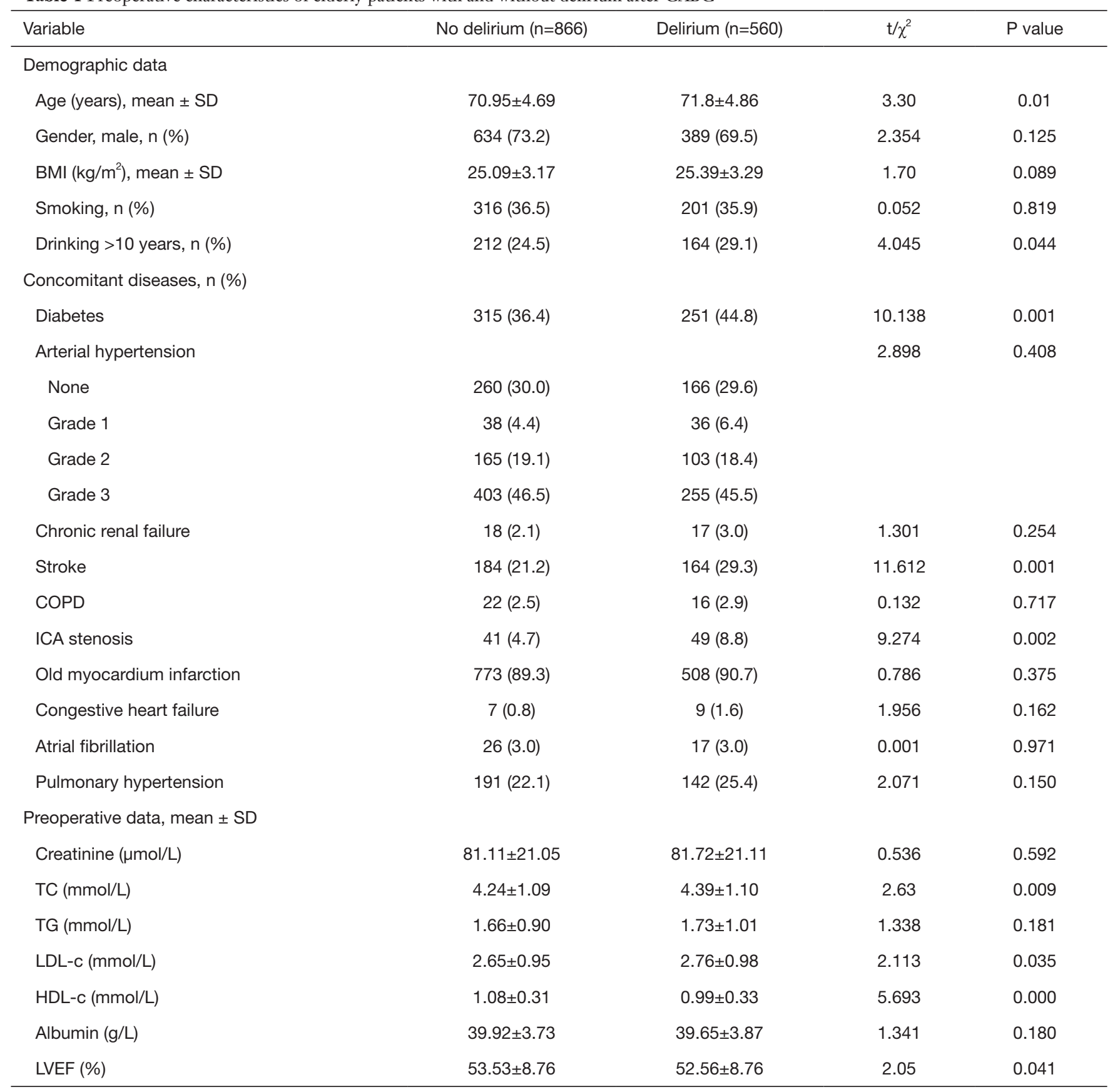

CABG, coronary artery bypass grafting; SD, standard deviation; BMI, body mass index; COPD, chronic obstructive pulmonary disease; ICA, internal carotid artery; TC, total cholesterol; TG, triglycerides; LDL-c, low density lipoprotein cholesterol; HDL-c, high-density lipoprotein cholesterol; LVEF, left ventricular eject fraction.

logistic regression are illustrated in Figure 1; only the statistically significant results for the univariate analysis are shown. 7 independent risk factors for POD were verified. Age, long-term drinking, diabetes, stroke, ICA stenosis,
PMI, and prolonged ICU LOS were all independent risk factors that resulted in POD in elderly CABG patients, while higher preoperative serum HDL-C and postoperative $\mathrm{PO}_{2}$ levels were protective factors $(\mathrm{P}<0.05)$. 
Table 2 Patients' intraoperative and postoperative characteristics

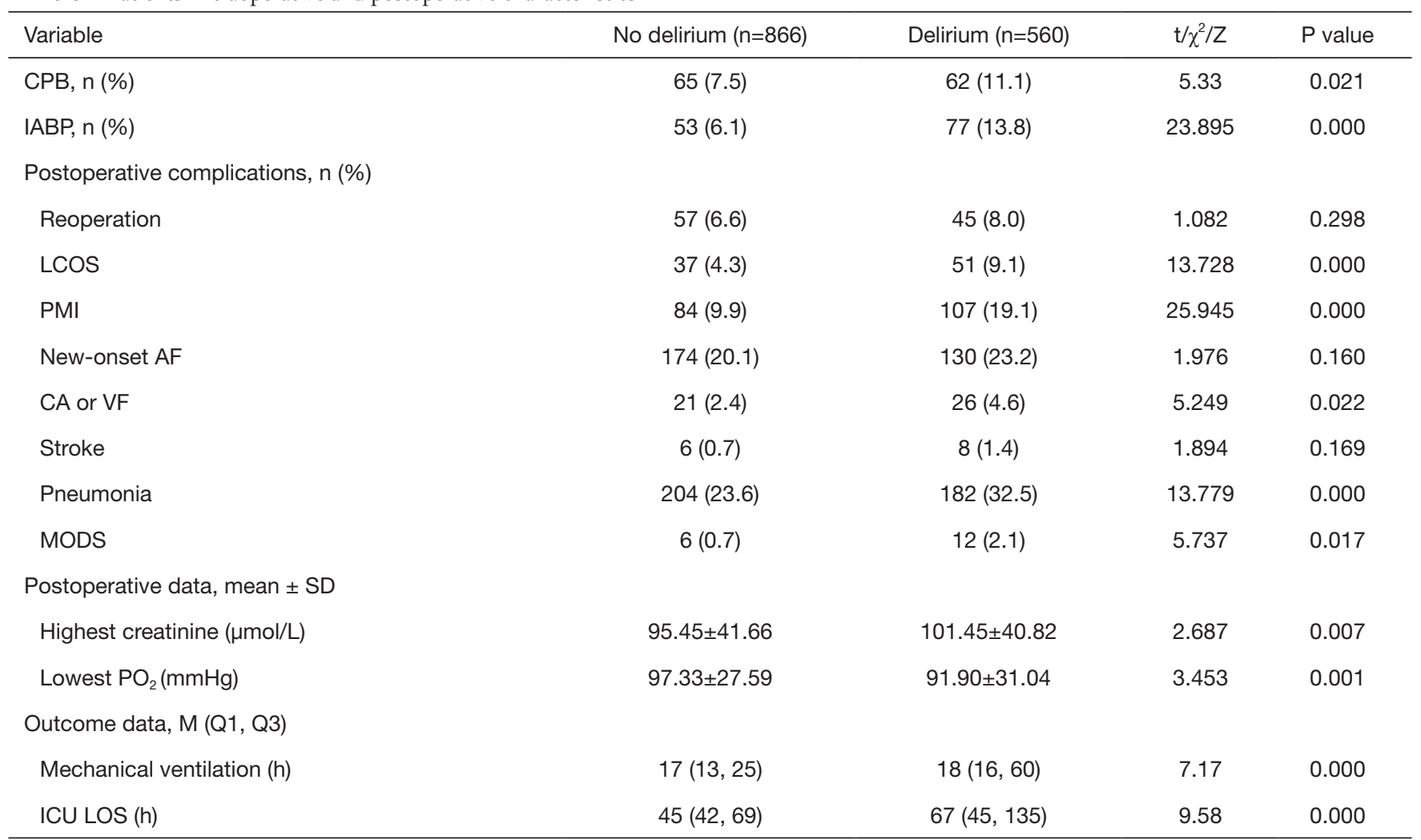

CPB, cardiopulmonary bypass; IABP, intra-aortic balloon pump; AF, atrial fibrillation; LCOS, low cardiac output syndrome; PMI, perioperative myocardial infarction; AF, atrial fibrillation; CA, cardiac arrest; VF, ventricular fibrillation; MODS, multiple organ dysfunction syndrome; $\mathrm{SD}$, standard deviation; $\mathrm{PO}_{2}$, arterial oxygen partial; $\mathrm{M}$, median; $\mathrm{Q} 1$, first quartile; $\mathrm{Q} 3$, third quartile; ICU, intensive care unit; LOS, length of stay.

Table 3 The grouping method and basis for continuous variables transformed into classified variables

\begin{tabular}{lcl}
\hline Variable & Cut-off value & \multicolumn{1}{c}{ Basis } \\
\hline HDL-C $(\mathrm{mmol} / \mathrm{L})$ & 1.0 & $\begin{array}{l}\text { The appropriate levels and abnormal stratified standards of blood lipids for the } \\
\text { primary prevention population of ASCVD in China (18) }\end{array}$ \\
Postoperative creatinine $(\mu \mathrm{mol} / \mathrm{L})$ & 88.5 & Median \\
Postoperative $\mathrm{PO}_{2}(\mathrm{mmHg})$ & 80 & Normal reference values \\
ICU LOS (hours) & 48 & The average level of our center \\
Mechanical ventilation time (hours) & 17 & Median \\
\hline
\end{tabular}

$\mathrm{HDL}-\mathrm{C}$, high-density lipoprotein cholesterol; ASCVD, atherosclerotic cardiovascular disease; $\mathrm{PO}_{2}$, arterial partial pressure of oxygen; ICU, intensive care unit; LOS, length of stay.

\section{Propensity score-matched analysis}

A propensity score-matched analysis was conducted based on the preoperative factors to modulate for potential confounding variables and selection bias. There were 1,426 (560 with POD and 866 without POD) complete cases eligible for matching. Of these, 1,012 (506 with POD and 506 without POD) were matched (see Table 4). Figure 2 presents the distribution of propensity scores for each group before PSM. The distributions were similar after PSM, indicating high-quality matching. In addition, none of the covariates differed significantly between the groups after 
Table 4 Preoperative patient characteristics after PSM

\begin{tabular}{|c|c|c|c|c|}
\hline Variable & \multicolumn{2}{|c|}{ Propensity-matched patients $(n=1,012)$} & $P$ value & Standard difference \\
\hline Age (years), mean \pm SD & $71.26 \pm 4.71$ & $71.39 \pm 4.56$ & 0.665 & 0.027 \\
\hline Gender, male, n (\%) & $148 \pm 29.2$ & $142 \pm 28.1$ & 0.728 & 0.026 \\
\hline BMI $\left(\mathrm{kg} / \mathrm{m}^{2}\right), \mathrm{n}(\%)$ & & & 0.850 & 0.056 \\
\hline $18.5-24$ & $169(33.4)$ & $171(33.8)$ & & \\
\hline $24-28$ & $241(47.6)$ & $248(49.0)$ & & \\
\hline$>28$ & $92(18.2)$ & $82(16.2)$ & & \\
\hline Smoking, n (\%) & $171(33.8)$ & $178(35.2)$ & 0.692 & 0.029 \\
\hline \multicolumn{5}{|l|}{ Concomitant diseases, n (\%) } \\
\hline Diabetes & $221(43.7)$ & $218(43.1)$ & 0.899 & 0.012 \\
\hline Arterial hypertension & & & 0.811 & 0.062 \\
\hline None & $145(28.7)$ & $156(30.8)$ & & \\
\hline Grade 1 & $29(5.7)$ & $32(6.3)$ & & \\
\hline Grade 2 & $100(19.8)$ & $92(18.2)$ & & \\
\hline Grade 3 & $232(45.8)$ & $226(44.7)$ & & \\
\hline Chronic renal failure & $14(2.8)$ & $14(2.8)$ & 1.000 & $<0.001$ \\
\hline Stroke & $142(28.1)$ & $136(26.9)$ & 0.725 & 0.027 \\
\hline Pulmonary hypertension & $118(23.3)$ & $120(23.7)$ & 0.941 & 0.009 \\
\hline \multicolumn{5}{|l|}{ Preoperative data, mean \pm SD } \\
\hline Creatinine $(\mu \mathrm{mol} / \mathrm{L})$ & $81.08 \pm 21.81$ & $81.14 \pm 20.16$ & 0.964 & 0.003 \\
\hline $\mathrm{TC}(\mathrm{mmol} / \mathrm{L})$ & $101 \pm 20.0$ & $95 \pm 18.8$ & 0.691 & 0.030 \\
\hline $\mathrm{TG}(\mathrm{mmol} / \mathrm{L})$ & $260 \pm 51.4$ & $256 \pm 50.6$ & 0.850 & 0.016 \\
\hline LDL-c (mmol/L) & $265 \pm 52.4$ & $263 \pm 52.0$ & 0.950 & 0.008 \\
\hline HDL-c (mmol/L) & $1.76 \pm 0.99$ & $1.74 \pm 1.03$ & 0.768 & 0.019 \\
\hline Albumin (g/L) & $39.73 \pm 3.86$ & $39.76 \pm 3.77$ & 0.874 & 0.010 \\
\hline LVEF (\%) & $52.79 \pm 8.68$ & $52.72 \pm 8.78$ & 0.903 & 0.008 \\
\hline
\end{tabular}

PSM, propensity-score matching; SD, standard deviation; BMI, body mass index; COPD, chronic obstructive pulmonary disease; ICA, internal carotid artery; TC, total cholesterol; TG, triglycerides; LDL-c, low density lipoprotein cholesterol; HDL-c, high-density lipoprotein cholesterol; LVEF, left ventricular eject fraction. 
Univariate analysis

\begin{tabular}{|c|c|c|c|c|}
\hline Characteristic & Odds Ratio $(95 \% \mathrm{Cl})$ & $P$ Value & & \\
\hline Age (years) & $1.04(1.01-1.06)$ & 0.001 & 5 & \\
\hline Drinking $>10$ years & $1.28(1.01-1.62)$ & 0.045 & $\infty-1$ & \\
\hline \multicolumn{5}{|c|}{ Concomitant diseases } \\
\hline Diabetes & $1.42(1.14-1.76)$ & 0.001 & $\mapsto-1$ & \\
\hline Strokes & $1.54(1.20-1.96)$ & 0.001 & $1 \mapsto-1$ & \\
\hline ICA stenosis & $1.93(1.26-2.96)$ & 0.003 & $\longmapsto$ & \\
\hline \multicolumn{5}{|l|}{ Preoperative data } \\
\hline $\mathrm{HDL}-\mathrm{C} \geq 1.0 \mathrm{mmo} / \mathrm{L}$ & $0.73(0.59-0.90)$ & 0.003 & & \\
\hline EF\% & $0.99(0.98-1.00)$ & 0.041 & & \\
\hline \multicolumn{5}{|c|}{ Intraoperative and postoperative factors } \\
\hline $\mathrm{CPB}$ & $1.53(1.06-2.21)$ & 0.022 & $\mapsto$ & \\
\hline IABP & $2.45(1.69-3.53)$ & $<0.001$ & $\longmapsto$ & \\
\hline $\mathrm{Cr} \geq 88.5 \mu \mathrm{mol} / \mathrm{L}$ & $1.37(1.11-1.70)$ & 0.003 & $1+-1$ & \\
\hline $\mathrm{PO} 2 \geq 80 \mathrm{mmHg}$ & $0.51(0.40-0.65)$ & $<0.001 \quad|9|$ & & \\
\hline Lcos & $2.24(1.45-3.48)$ & $<0.001$ & $\longmapsto$ & \\
\hline PMI & $2.20(1.62-2.99)$ & $<0.001$ & $\longmapsto \quad 1$ & \\
\hline CA or VF & $1.96(1.09-3.52)$ & 0.024 & $\longrightarrow$ & \\
\hline Pneumonia & $1.56(1.23-1.98)$ & $<0.001$ & $1 \bullet-1$ & \\
\hline MODS & $3.14(1.17-8.41)$ & 0.023 & $\longmapsto$ & $\rightarrow$ \\
\hline ICU LOS $>48 \mathrm{~h}$ & $2.05(1.65-2.55)$ & $<0.001$ & $\mapsto$ & \\
\hline PWV & $1.85(1.49-2.30)$ & $<0.001$ & $\mapsto-1$ & \\
\hline
\end{tabular}

Multivariate analysis

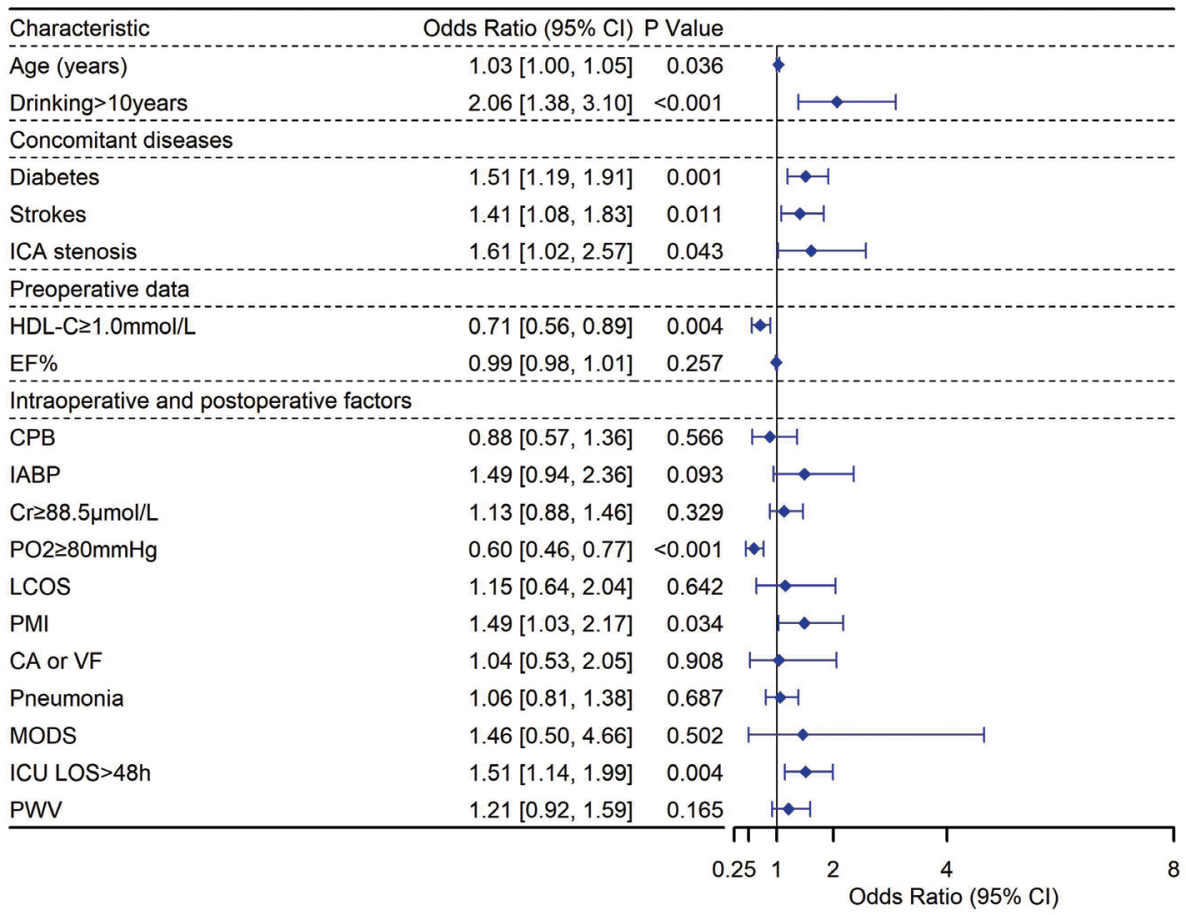

Figure 1 Univariate regression analysis and multivariable regression analysis for patients with delirium. CI, confidence interval; ICA, internal carotid artery; HDL-C, high-density lipoprotein cholesterol; EF, ejection fraction; CPB, cardiopulmonary bypass; IABP, intra-aortic balloon pump; Cr, creatinine; PO2, arterial oxygen partial; LCOS, low cardiac output syndrome; PMI, perioperative myocardial infarction; CA, cardiac arrest; VF, ventricular fibrillation; MODS, multiple organ dysfunction syndrome; ICU, intensive care unit; LOS, length of stay; PMV, prolonged mechanical ventilation. 
PSM (see Table 4). The multivariate regression analysis following PSM showed that prolonged ICU stay remained an independent risk factor for POD, but PMI and higher $\mathrm{PO}_{2}$ levels were no longer significantly associated with delirium (see Table 5).

Table 5 Multivariate regression analysis based on PSM for operative and postoperative factors influencing the risk of delirium

\begin{tabular}{|c|c|c|}
\hline \multirow{2}{*}{ Variables } & \multicolumn{2}{|c|}{ Matched patients $(n=1,012)$} \\
\hline & Odds ratio $(95 \% \mathrm{Cl})$ & $P$ value \\
\hline Cardiopulmonary bypass & $1.04(0.64,1.69)$ & 0.871 \\
\hline Intra-aortic balloon pump & $0.60(0.36,1.01)$ & 0.056 \\
\hline Creatinine & $0.89(0.68,1.16)$ & 0.399 \\
\hline Arterial oxygen partial & $1.15(0.87,1.53)$ & 0.317 \\
\hline Low cardiac output syndrome & $1.63(0.85,3.18)$ & 0.149 \\
\hline $\begin{array}{l}\text { Perioperative myocardial } \\
\text { infarction }\end{array}$ & $1.23(0.82,1.85)$ & 0.329 \\
\hline $\begin{array}{l}\text { Cardiac arrest or ventricular } \\
\text { fibrillation }\end{array}$ & $0.69(0.32,1.50)$ & 0.35 \\
\hline Pneumonia & $1.11(0.82,1.49)$ & 0.506 \\
\hline $\begin{array}{l}\text { Multiple organ dysfunction } \\
\text { syndrome }\end{array}$ & $1.15(0.34,3.97)$ & 0.824 \\
\hline Prolonged ICU LOS & $1.62(1.19,2.21)$ & 0.002 \\
\hline $\begin{array}{l}\text { Prolonged mechanical } \\
\text { ventilation }\end{array}$ & $1.17(0.87,1.58)$ & 0.304 \\
\hline
\end{tabular}

PSM, propensity-score matching; ICU, intensive care unit; LOS, length of stay; $\mathrm{Cl}$, confidence interval.

\section{Discussion}

Age has a significant effect on the development of POD and may be a vicarious variable for the accumulation of agerelated risk factors that are expressed differently among individuals. The sum of these risk factors is the most important factor in determining the occurrence of POD (2). The rate of delirium is heavily influenced by the method of diagnosis (7). The CAM-ICU is easily applicable, can be more rapidly performed than a diagnosis using current reference standards, including the Diagnostic and Statistical Manual of Mental Disorder-5, and has high specificity and sensitivity (19). In this study, we used the CAM-ICU as a standardized diagnostic instrument for POD in elderly patients who underwent CABG, and found an incidence rate of $39.3 \%$.It was slightly higher than the incidence of delirium after CABG in other studies $(7,14)$, which may be related to different included populations, higher average age of the patients included in our study.

The risk factors of POD in CABG were a focus of clinical research. Many predictors have been reported by researchers $(14,16,17)$, including age, preoperative cognitive impairment and concomitant diseases, ventilator time, ICU stay time, etc, most of these factors were non-modifiable. In this study, we pay more attention to some modifiable factors, such as smoking, drinking, preoperative serum lipid level and nutritional status, postoperative hypoxemia. These factors may have more clinical significance in decreasing the occurrence of delirium. A total of 36 factors were included in the analysis. The multivariate logistic regression analysis of all patients showed that age, long-term drinking, diabetes,
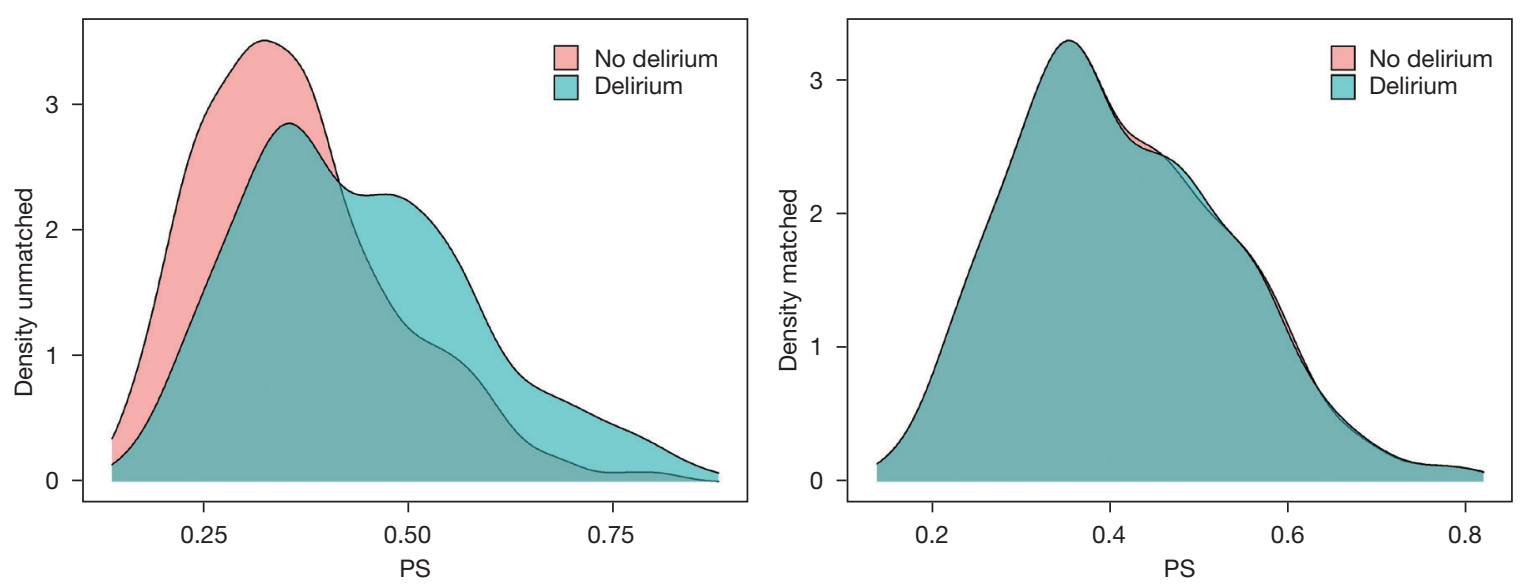

Figure 2 Density plot of the logit of the propensity score before and after PSM. PS, propensity score; PSM, propensity-score matching. 
stroke, carotid artery stenosis, PMI, and a prolonged ICU stay were independent risk factors for POD. Preoperative HDL-C levels of $\geq 1.0 \mathrm{mmol} / \mathrm{L}$ and postoperative oxygen partial pressure of $\geq 80 \mathrm{mmHg}$ were protective factors for preventing POD. A number of factors were included in our research, specifically preoperative characteristics, on-pump or off-pump CABG, and postoperative complications. Some factors may influence each other; for example, intraoperative management and perioperative complications will be affected by preoperative complications and a patient's baseline health status. Thus, we used PSM to balance the preoperative confounding factors, and analyzed the effects of cardiopulmonary bypass and postoperative factors on POD. When confounding factors were excluded, the results showed that a prolonged ICU stay was an independent risk factor for POD; however, the exclusion of confounding factors, PMI, and postoperative arterial oxygen partial pressure had no effect on the results.

Older age is a predictor for POD in the general population after cardiac surgery (20-22). In general, patients with lower cognitive function and organ function reserve have a decreased ability to maintain normal brain function when under perioperative stress. Many studies have shown that the underlying risk of delirium increases with the presence of diabetes, stroke, peripheral vascular stenosis, and other underlying diseases $(16,17)$. The results of our study are consistent with those of previous studies. The independent risk factors for delirium following CABG in elderly patients are age, diabetes, stroke, and carotid artery stenosis.

The regular intake of alcohol can induce the release of neurotransmitters, such as dopamine, pentahydroxy tryptophan, opioid, and norepinephrine, in the brain, activate the mechanism of reinforcement and reward, and thus produce a dependence on alcohol (23). Brain receptors interact with alcohol to inhibit excitatory nerve pathway activity and interfere with communication between nerve cells. Neurodegeneration, neurocognitive deficits, and neuronal injury are well documented in alcoholics; thus, chronic excessive drinking can cause serious problems with memory and cognition (24). In this study, we extensively analyzed the effect of long-term drinking ( $>10$ years and continued to the operation) on POD in elderly patients undergoing $\mathrm{CABG}$, and found that the proportion of patients with a history of long-term drinking in the delirium group was visibly higher than that in the non-delirium group $(\mathrm{P}=0.044)$. The multivariate logistic regression analysis showed that long-term drinking was identified as an independent risk factor for POD $(\mathrm{P}=0.004$, or $\mathrm{P}=1.447)$. Preoperative guidance directing patients to stop drinking and abstain from alcohol intake is important in preventing POD.

HDL-C can reverse the transport of cholesterol from peripheral tissues, such as the blood vessel wall, allowing it to undergo catabolism in the liver, and thus reduce the deposition of cholesterol in the blood vessel wall by playing an anti-atherosclerotic role. The results of the univariate analysis showed that the preoperative serum HDL-C level in the non-delirium group was significantly higher than that in the delirium group $(\mathrm{P}<0.01)$. Under the 2016 Chinese guidelines for dyslipidemia management in adults, the cutoff point for the serum HDL-C level is $1.0 \mathrm{mmol} / \mathrm{L}$. Our multivariate regression analysis showed that a preoperative HDL-C level $\geq 1.0$ was a protective factor for POD. It may be that HDL-C has a reversal effect, which can reduce lipid deposition and maintain the stability of the normal bloodbrain barrier, thus inhibiting the diffusion of carbon dioxide outside the cerebral vessels and causing damage to glial cells and neurons.

Some limitations of this study should be noted. One of the most important limitations is the performance of the study in a single-center cohort may not be extrapolated to the cardiac surgery population at large nor in another center. The interpretation of the data is limited because of limited number of patients, and a possible selection bias. In addition, the absence of full knowledge about other possible delirium risk factors, could lead to some inaccuracies. So, large-scale multi center research is desired in the future.

\section{Conclusions}

In conclusion, the morbidity of POD in elderly patients undergoing $C A B G$ is high. The occurrence of delirium is influenced by age-related cumulative factors. Age, longterm alcohol consumption, diabetes, stroke, and carotid artery stenosis were independent risk factors for delirium in elderly patients who underwent CABG. After adjusting for preoperative confounding factors with PSM, it was concluded that among the postoperative factors, only ICU stay was an independent risk factor for POD. Additionally, a higher HDL-C level was shown to be a potential protective factor in preventing POD.

\section{Acknowledgments}

We would like to express our gratitude to the patients who 
provided the data and the staff at the Department of Cardiac Surgery and the Department of Anesthesiology, Intensive Care Unit at the Tianjin Chest Hospital in Tianjin, China. Funding: None.

\section{Footnote}

Reporting Checklist: The authors have completed the STROBE reporting checklist. Available at https://dx.doi. org/10.21037/atm-21-5160

Data Sharing Statement: Available at https://dx.doi. org/10.21037/atm-21-5160

Conflicts of Interest: All authors have completed the ICMJE uniform disclosure form (available at https://dx.doi. org/10.21037/atm-21-5160). The authors have no conflicts of interest to declare.

Etbical Statement: The authors are accountable for all aspects of the work in ensuring that questions related to the accuracy or integrity of any part of the work are appropriately investigated and resolved. The study was performed in accordance with the Declaration of Helsinki (as revised in 2013). The study was approved by the Ethics Committee of Tianjin Chest Hospital (No. 2021LW-012). The requirement of personal consent for this retrospective analysis was waived. To ensure patient confidentiality, the analyses were performed on de-identified data.

Open Access Statement: This is an Open Access article distributed in accordance with the Creative Commons Attribution-NonCommercial-NoDerivs 4.0 International License (CC BY-NC-ND 4.0), which permits the noncommercial replication and distribution of the article with the strict proviso that no changes or edits are made and the original work is properly cited (including links to both the formal publication through the relevant DOI and the license). See: https://creativecommons.org/licenses/by-nc-nd/4.0/.

\section{References}

1. Association AP. Diagnostic and Statistical Manual of Mental Disorders. 5th ed. Washington, DC: American Psychiatric Association, 2013.

2. Aldecoa C, Bettelli G, Bilotta F, et al. European Society of Anaesthesiology evidence-based and consensus-based guideline on postoperative delirium. Eur J Anaesthesiol
2017;34:192-214.

3. Vasilevskis EE, Han JH, Hughes CG, et al. Epidemiology and risk factors for delirium across hospital settings. Best Pract Res Clin Anaesthesiol 2012;26:277-87.

4. Head SJ, Milojevic M, Taggart DP, et al. Current Practice of State-of-the-Art Surgical Coronary Revascularization. Circulation 2017;136:1331-45.

5. Melly L, Torregrossa G, Lee T, et al. Fifty years of coronary artery bypass grafting. J Thorac Dis 2018;10:1960-7.

6. Head SJ, Milojevic M, Daemen J, et al. Mortality after coronary artery bypass grafting versus percutaneous coronary intervention with stenting for coronary artery disease: a pooled analysis of individual patient data. Lancet 2018;391:939-48.

7. Greaves D, Psaltis PJ, Ross TJ, et al. Cognitive outcomes following coronary artery bypass grafting: A systematic review and meta-analysis of 91,829 patients. Int J Cardiol 2019;289:43-9.

8. Lechowicz K, Szylińska A, Listewnik M, et al. Cardiac Delirium Index for Predicting the Occurrence of Postoperative Delirium in Adult Patients After Coronary Artery Bypass Grafting. Clin Interv Aging 2021;16:487-95.

9. Gottesman RF, Grega MA, Bailey MM, et al. Delirium after coronary artery bypass graft surgery and late mortality. Ann Neurol 2010;67:338-44.

10. Santos FS, Velasco IT, Fráguas R Jr. Risk factors for delirium in the elderly after coronary artery bypass graft surgery. Int Psychogeriatr 2004;16:175-93.

11. van Munster BC, Korevaar JC, Zwinderman AH, et al. The association between delirium and the apolipoprotein E epsilon 4 allele: new study results and a meta-analysis. Am J Geriatr Psychiatry 2009;17:856-62.

12. Adamis D, Meagher D, Williams J, et al. A systematic review and meta-analysis of the association between the apolipoprotein E genotype and delirium. Psychiatr Genet 2016;26:53-9.

13. Sepulveda E, Adamis D, Franco JG, et al. The complex interaction of genetics and delirium: a systematic review and meta-analysis. Eur Arch Psychiatry Clin Neurosci 2021;271:929-39.

14. Zhang WY, Wu WL, Gu JJ, et al. Risk factors for postoperative delirium in patients after coronary artery bypass grafting: A prospective cohort study. J Crit Care 2015;30:606-12.

15. Oldham MA, Hawkins KA, Lin IH, et al. Depression Predicts Delirium After Coronary Artery Bypass 
Graft Surgery Independent of Cognitive Impairment and Cerebrovascular Disease: An Analysis of the Neuropsychiatric Outcomes After Heart Surgery Study. Am J Geriatr Psychiatry 2019;27:476-86.

16. Kotfis K, Szylińska A, Listewnik M, et al. Early delirium after cardiac surgery: an analysis of incidence and risk factors in elderly ( $\geq 65$ years) and very elderly ( $\geq 80$ years) patients. Clin Interv Aging 2018;13:1061-70.

17. Greaves D, Psaltis PJ, Davis DHJ, et al. Risk Factors for Delirium and Cognitive Decline Following Coronary Artery Bypass Grafting Surgery: A Systematic Review and Meta-Analysis. J Am Heart Assoc 2020;9:e017275.

18. Joint committee for guideline revision. 2016 Chinese guidelines for the management of dyslipidemia in adults. J Geriatr Cardiol 2018;15:1-29.

19. Chen TJ, Chung YW, Chang HR, et al. Diagnostic accuracy of the CAM-ICU and ICDSC in detecting

Cite this article as: Li J, Meng D, Chang C, Fu B, Xie C, Wu Z, Wang L. Risk factors for delirium after coronary artery bypass grafting in elderly patients. Ann Transl Med 2021;9(22):1666. doi: $10.21037 / \mathrm{atm}-21-5160$ intensive care unit delirium: A bivariate meta-analysis. Int J Nurs Stud 2021;113:103782.

20. Tan MC, Felde A, Kuskowski M, et al. Incidence and predictors of post-cardiotomy delirium. Am J Geriatr Psychiatry 2008; 16:575-83.

21. Koster S, Hensens AG, van der Palen J. The long-term cognitive and functional outcomes of postoperative delirium after cardiac surgery. Ann Thorac Surg 2009;87:1469-74.

22. Inouye SK, Westendorp RG, Saczynski JS. Delirium in elderly people. Lancet 2014;383:911-22.

23. Michalak A, Biała G. Alcohol dependence--neurobiology and treatment. Acta Pol Pharm 2016;73:3-12.

24. Mukherjee S. Alcoholism and its effects on the central nervous system. Curr Neurovasc Res 2013;10:256-62.

(English Language Editor: L. Huleatt) 